\title{
THE PARTNERSHIP OF POTENTIALS IN QUANTUM MECHANICS AND SHAPE INVARIANCE*
}

\author{
JOSÉ F. CARIÑNAA ${ }^{\dagger}$ and ARTURO RAMOS ${ }^{\ddagger}$ \\ Departamento de Física Teórica, Facultad de Ciencias, \\ Universidad de Zaragoza, 50009-Zaragoza, Spain
}

\begin{abstract}
The concept of partnership of potentials is studied in detail and in particular the nonuniqueness due to the ambiguity in the election of the factorization energy and in the choice of the solution of certain Riccati equation. We generate new factorizations from old ones using invariance under parameter transformations. The theory is illustrated with some examples.
\end{abstract}

Keywords: Factorization Method, partner potentials, intertwining technique, Shape Invariance.

\section{Introduction}

The so-called Factorization Method plays an important role in the search for quantum systems for which the spectrum of the Hamiltonian operators is completely known. 晴 It is closely related with the existence of an intertwining operator, with Supersummetric Quantum Mechanics 6 and Darboux transformations in this last context. Moreover, these techniques have important generalizations to higher dimensional spaces 10 to higher order factorization operators., $10,11.12$ and to the class of systems with partial algebraization of the spectrum, $13,14,15$ among others. Actually, most exactly solvable potentials can be obtained by making use of an appropriate intertwining operator transformation.

Looking for a factorization of a given Hamiltonian amounts to find a constant $d$ and a solution of a Riccati differential equation for the superpotential function. Once a solution has been found, a partner potential is defined such that it has almost the same spectrum as the original one. However, the point is that with a different choice for the solution of the mentioned Riccati equation, a different partner potential is obtained. This fact has been shown to be very useful for the search of isospectral potentials, an idea due to Mielnik 16 and later developed in other articles.17. $18.19,20$

\footnotetext{
*To appear in Modern Physics Letters A

$\dagger$ E-mail: jfc@posta.unizar.es

${ }^{\ddagger}$ E-mail: arrg@posta.unizar.es
} 
Moreover, the ambiguity in the definition of a partner potential of a given one is twofold: firstly, due to the choice of the factorization energy $d$, which is not unique in general, and then, it arises the ambiguity in the election of the solution of the corresponding Riccati equation. These two ambiguities are more or less known and are implicitly used or mentioned in many papers. However, we feel that it is worth having a new look at the subject in its own right because its understanding allows to interpret certain facts treated in the literature as consequences of this undetermination. In addition, these ideas have a great influence in the same concept of partnership when applied to the subclass of Shape Invariant partner potentials.

Shape Invariance is an important concept in the theory of exactly solvable systems, which was explicitly introduced by Gendenshteïn,21 although the basic idea was already present, to some extent, in the classic work of Infeld and Hull. 1 That was suggested by some authors several years ago 2223 and has been shown with some detail recently.24 The ambiguity in the definition of the partner potential is inherited in the case of Shape Invariance, so one may wonder to what extent it makes sense the relation between a potential and its partner characterizing such a kind of problems.

Therefore, two main questions arise. Are there different solutions for the same Riccati equation leading to the same partner?. On the other hand, if the Shape Invariance condition holds for a certain partner, is it also true for any other possible partner?. Our aim is to analyze these questions using, among other things, the machinery for dealing with Riccati equations developed in previous papers.24 25

The letter is organized as follows. In the next section we will review briefly the concepts of partner potential, when one potential is given, and Shape Invariance.

In Section 3 we look at the abovementioned ambiguities. In two recent papers 2620 two alternative factorizations for different choices of the constant $d$ are given. We will analyze this point in Section 4, showing that the ambiguity in the factorization energy, and hence the existence of some factorizations is due, in turn, to certain parameter invariance symmetry of the given potential. Moreover, if the given potential can be considered as being part of a pair of Shape Invariant partner potentials, more factorizations can be found. We develop these ideas with some illustrative examples.

\section{Factorization method and shape invariance}

The simplest way of generating a new exactly solvable Hamiltonian $\widetilde{H}$ from a known one $H$ is just to consider an invertible bounded operator $B$, with bounded inverse, and defining $\widetilde{H}=B H B^{-1}$. This new Hamiltonian $\widetilde{H}$ has the same spectrum as the starting Hamiltonian $H$. As a generalization, 3 we will say that two Hamiltonian operators $H$ and $\widetilde{H}$ are $A$-related when $A H=\widetilde{H} A$, where $A$ may be singular. In this case, if $\psi$ is an eigenvector of $H$ corresponding to the eigenvalue $E$ and $A \psi \neq 0$, then, at least formally, $A \psi$ is also an eigenvector of $\widetilde{H}$ corresponding to the same eigenvalue $E$. 
If we assume that the intertwining operator $A$ is a first order differential operator,

$$
A=\frac{d}{d x}+W(x), \quad \text { and } \quad A^{\dagger}=-\frac{d}{d x}+W(x),
$$

then the relation $A H=\widetilde{H} A$, with

$$
H=-\frac{d^{2}}{d x^{2}}+V(x), \quad \widetilde{H}=-\frac{d^{2}}{d x^{2}}+\widetilde{V}(x),
$$

leads to

$$
V=-2 W^{\prime}+\tilde{V}, \quad W(V-\widetilde{V})=-W^{\prime \prime}-V^{\prime},
$$

and taking into account the first equation, the second one becomes $2 W W^{\prime}=W^{\prime \prime}+$ $V^{\prime}$, which can easily be integrated giving

$$
\begin{aligned}
& V=W^{2}-W^{\prime}+d, \\
& \widetilde{V}=W^{2}+W^{\prime}+d,
\end{aligned}
$$

where $d$ is an integration constant. The important point here is that $H$ and $\widetilde{H}$, given by (1), can be related by a first order differential operator $A$ of the form given above if, and only if, there exists a constant $d$ and a function $W$ such that the pair of Riccati equations (2) and (3) are satisfied simultaneously. Moreover, this means that both Hamiltonians can be factorized as

$$
H=A^{\dagger} A+d, \quad \widetilde{H}=A A^{\dagger}+d .
$$

Adding and subtracting equations (2) and (3) we obtain the equivalent pair which relates $V$ and $\widetilde{V}$

$$
\begin{aligned}
\widetilde{V}-d & =-(V-d)+2 W^{2} \\
\widetilde{V} & =V+2 W^{\prime} .
\end{aligned}
$$

The function $W$ satisfying these equations is usually called superpotential, the constant $d$ factorization energy or factorization constant and $\widetilde{V}$ and $V$ (resp. $\widetilde{H}$ and $H)$ are said to be partner potentials (resp. Hamiltonians).

Gendenshteïn took equations (2) and (3) as a definition of the functions $V$, $\widetilde{V}$ in terms of the function $W$ and some constant $d$. After, he supposed that $W$ did depend on certain set of parameters $a, W=W(x, a)$, and as a consequence $V=V(x, a)$ and $\widetilde{V}=\widetilde{V}(x, a)$ as well. Then, the necessary condition for $\widetilde{V}(x, a)$ to be of the same form as $V(x, a)$, maybe for a different choice of the values of the parameters involved in $V$, is known as Shape Invariance. More explicitly, it amounts to assume the further relation between $V(x, a)$ and $\widetilde{V}(x, a)$

$$
\widetilde{V}(x, a)=V(x, f(a))+R(f(a)),
$$

where $f$ is an (invertible) transformation on the parameter space $a$ and $R$ is some function. The main advantage of these problems is that the complete spectrum of 
the corresponding Hamiltonians $H$ and $\widetilde{H}$ can be found easily 21 Let us remark that it is the choice of $a$ and $f(a)$ what defines the different classes of Shape Invariant potentials. The function $f$ may be even the identity, $f(a)=a .22$ For a more detailed information see, for example, Ref. 24 .

\section{On the ambiguity in the definition of the partner potential}

Given one potential function $V$, the equation (2) to be solved when searching for a superpotential function $W$, once $d$ is fixed, is a Riccati equation. In general, its general solution cannot be found by means of quadratures. However, now we only need to compare solutions of the same equation when a particular solution is known. In such a case, it is well known that its general solution can be written using two quadratures. For a group theoretical explanation of this fact, see, for example, Ref. 25. Our aim now is to study a bit further the general solution of (2) in that situation and analyze the corresponding possible partner potentials $\widetilde{V}$.

It is well known 2425 that if $W_{p}$ is a particular solution of (2) for some specific constant $d$, the change of variable

$$
v=\frac{1}{W_{p}-W}, \quad \text { with inverse } \quad W=W_{p}-\frac{1}{v},
$$

transforms (2) into the inhomogeneous first order linear equation for $v$

$$
\frac{d v}{d x}=-2 W_{p} v+1
$$

which has the general solution

$$
v(x)=\frac{\int^{x} \exp \left\{2 \int^{\xi} W_{p}(\eta) d \eta\right\} d \xi+F}{\exp \left\{2 \int^{x} W_{p}(\xi) d \xi\right\}},
$$

where $F$ is an integration constant. Therefore, the general solution of (2) reads as

$$
W_{g}(x)=W_{p}(x)-\frac{\exp \left\{2 \int^{x} W_{p}(\xi) d \xi\right\}}{\int^{x} \exp \left\{2 \int^{\xi} W_{p}(\eta) d \eta\right\} d \xi+F} .
$$

We will review now the concept of partnership given one potential $V$. We have to find a constant $d$ and at least one particular solution $W_{p}$ of the Riccati equation (2). Then, "the partner" $\widetilde{V}$ is constructed by using (3) or equivalently (6). But these formulas explicitly show that $\widetilde{V}$ does depend upon the choice of the particular solution of (2) considered. Since the general solution of (2) can be written as $W_{g}=W_{p}-1 / v$, where $v$ is given by (10), the general solution obtained for $\widetilde{V}_{g}$ is, according to (6),

$$
\widetilde{V}_{g}=\widetilde{V}_{p}-2 \frac{d}{d x}\left(\frac{1}{v}\right)
$$

This answers one of the questions in the introduction: all the partner potentials, obtained by using (12) are different, apart from the trivial case in which $W_{p}$ and $V$ 
are constant, because the differential equation (9) only admits a constant solution when $W_{p}$ is constant.

This implies that "the partner" of one given potential is not a well defined concept and it seems better to say that an ordered pair $(V, \widetilde{V})$ is a supersymmetric pair of partner potentials if there exists a constant $d$ and a function $W$ such that this last is a common solution of the Riccati equations (2) and (3) constructed with these potentials, respectively. Of course the preceding comment shows that in such a case the superpotential function $W$ is essentially unique for each $d$, which moreover makes the problem of $A$-related Hamiltonians be well defined. Note as well that this reformulation of partnership comprehends the situation where $V$ is the potential we have started this section with, $\widetilde{V}$ is one of the functions obtained from (12) for a specific value of the constant $F$, and $W$ is obtained from (11) for the same value of $F$.

Now we will show what consequences have this undetermination in the subclass of Shape Invariant potentials. For that, we should use instead of (2) and (3) the equations

$$
\begin{aligned}
& V(x, a)-d(a)=W^{2}(x, a)-W^{\prime}(x, a), \\
& \widetilde{V}(x, a)-d(a)=W^{2}(x, a)+W^{\prime}(x, a),
\end{aligned}
$$

where now the factorization constant depends on the parameter a (see Ref. 24, Sec. 3 for details). Consider a particular solution $W_{p}(x, a)$ of equation (13) for some specific constant $d(a)$, such that it is also a particular solution of (14), being $V(x, a)$ and $\widetilde{V}(x, a)$ related by the further condition (7). As in the previous case, we can consider the general solution of (13) starting from $W_{p}(x, a)$, which is

$$
W_{g}(x, a, F)=W_{p}(x, a)+g(x, a, F),
$$

where $g(x, a, F)$ is defined by

$$
g(x, a, F)=-\frac{\exp \left\{2 \int^{x} W_{p}(\xi, a) d \xi\right\}}{\int^{x} \exp \left\{2 \int^{\xi} W_{p}(\eta, a) d \eta\right\} d \xi+F},
$$

being $F$ a integration constant. Note that the particular solution $W_{p}(x, a)$ is obtained from (15) as $F \rightarrow \infty$. Then, inserting $W_{g}(x, a, F)$ into an equation like (14) we obtain the general family of partner potentials

$$
\widetilde{V}(x, a, F)=\widetilde{V}(x, a)-2 g^{\prime}(x, a, F) .
$$

The question now is whether the condition (7) is maintained when we consider $\widetilde{V}(x, a, F)$ and $V(x, a)$ instead of $\widetilde{V}(x, a)$ and $V(x, a)$. Then, we ask for

$$
\widetilde{V}(x, a, F)=V(x, f(a))+\bar{R}(f(a), F),
$$

for some suitable $F$, where $f$ is the same as in $(7)$, and $\bar{R}(f(a), F)$ is a number not depending on $x$, maybe different from the $R(f(a))$ of (7). Taking into account (7) 
and (17), the equation (18) reads as

$$
2 g^{\prime}(x, a, F)=\bar{R}(f(a), F)-R(f(a)),
$$

that is, $2 g^{\prime}(x, a, F)$ should be a constant, which we name as $k$ for brevity. Integrating respect to $x$ we obtain $2 g(x, a, F)=k x+l$, being $l$ another constant depending at most on $a$ and $F$. Since $g(x, a, F)$ is, on the other hand, given by (16), it follows

$$
\int^{x} \exp \left\{2 \int^{\xi} W_{p}(\eta, a) d \eta\right\} d \xi+F=-\frac{2 \exp \left\{2 \int^{x} W_{p}(\xi, a) d \xi\right\}}{k x+l} .
$$

Differentiating this last equation, and solving for $W_{p}(x, a)$ we obtain

$$
W_{p}(x, k, l)=\frac{1}{4}\left(\frac{2 k}{k x+l}-(k x+l)\right),
$$

where we have made explicit that the parameter space should be $a=\{k, l\}$. Introducing this expression in (19) and performing the integrations, we obtain

$$
-2 e^{-x(k x+2 l) / 4}+F=-2 e^{-x(k x+2 l) / 4}
$$

and hence $F=0$. Now we have to check whether this particular case we have found, which is the only candidate for fulfilling (18), satisfy our hypothesis (7). The partner potentials defined by $W_{p}(x, k, l)$ and equations (13), (14) are

$$
\begin{aligned}
& V(x, k, l)-d(k, l)=W_{p}^{2}(x, k, l)-W_{p}^{\prime}(x, k, l)=\frac{(k x+l)^{2}}{16}+\frac{3 k^{2}}{4(k x+l)^{2}}, \\
& \widetilde{V}(x, k, l)-d(k, l)=W_{p}^{2}(x, k, l)+W_{p}^{\prime}(x, k, l)=\frac{(k x+l)^{2}}{16}-\frac{k^{2}}{4(k x+l)^{2}}-\frac{k}{2} .
\end{aligned}
$$

Now, we have to find out whether there are some transformation of the parameters $\{k, l\}$ such that the condition (7) be satisfied. Denoting the transformed parameters as $\left\{k_{1}, l_{1}\right\}$ for simplicity, we have

$$
\begin{gathered}
\widetilde{V}(x, k, l)-V\left(x, k_{1}, l_{1}\right)=d(k, l)-d\left(k_{1}, l_{1}\right)-\frac{k}{2}-\frac{1}{4}\left(\frac{3 k_{1}^{2}}{\left(k_{1} x+l_{1}\right)^{2}}+\frac{k^{2}}{(k x+l)^{2}}\right) \\
+\frac{1}{16}\left(\left(k-k_{1}\right) x+l-l_{1}\right)\left(\left(k+k_{1}\right) x+l+l_{1}\right) .
\end{gathered}
$$

The right hand side of this equation must be a constant and therefore, each of the different dependences on $x$ must vanish. The term $\left(\left(k-k_{1}\right) x+l-l_{1}\right)\left(\left(k+k_{1}\right) x+l+l_{1}\right)$ vanish for the combinations $k_{1}=-k, l_{1}=-l$ or $k_{1}=k, l_{1}=l$, apart form the case $k_{1}=-k_{1}=k=0$, which will be studied separately. However, the term

$$
\frac{3 k_{1}^{2}}{\left(k_{1} x+l_{1}\right)^{2}}+\frac{k^{2}}{(k x+l)^{2}}
$$

is equal to $4 k^{2} /(k x+l)^{2}$ for both combinations and does not vanish. Then, the Shape Invariance hypothesis is not satisfied. In the case of $k=0$ we have that the 
corresponding $W_{p}(x, a)$ is a constant and hence provides the trivial case where the corresponding partner potentials are constant as well.

This answers the other question of the introduction, and it is closely related with the previous one. That is, if the Shape Invariance condition holds for a possible partner, then it does not hold for any other choice of partner, apart from the trivial case where all the involved functions are constant.

As a consequence of all the previous, it would be better to reformulate the Shape Invariance condition (7) in terms of appropriate $W$ and $d$ only. Now, considering a particular common solution $W(x, a)$ of $(13)$ and (14) for some $d(a)$, jointly with (7) allows to write this last condition as

$$
W^{2}(x, a)-W^{2}(x, f(a))+W^{\prime}(x, f(a))+W^{\prime}(x, a)=R(f(a)),
$$

where $R(f(a))=d(f(a))-d(a)$. This way, beginning from $W(x, a)$ and $d(a)$ which solve (20) for some $f$, we will obtain through (13) and (14) well defined Shape Invariant partner potentials $(V(x, a), \widetilde{V}(x, a))$ by construction. In the celebrated article by Infeld and Hull the key point is indeed to solve an equation of type (20) (see their equation (3.1.2)). Similarly, in Ref. 27, Sec. VI, the main point in the classification of Shape Invariant potentials they propose is to find solutions of an equation of type (20) (see their formula (2.22)). An equation of type (20) also plays a central role in more recent articles, 2428 where some of the results of Infeld and Hull are reviewed and put in connection with the concept of Shape Invariance, and Shape Invariant partner potentials depending on $n$ parameters transformed by translation are found, giving a solution to a previously unsolved problem.27 However, it seems that a justification of why it is necessary to solve an equation of type (20) when searching for well defined Shape Invariant partner potentials has not been given explicitly in the literature up to now.

\section{Parameter invariance and Shape Invariance: existence of several fac- torizations}

We will analyze in this section what happens if there exists a transformation in the parameter space, $g: a \mapsto g(a)$ such that leaves the potential $V(x, a)$ in (13) invariant. Then, whenever $(W(x, a), d(a))$ is a solution of (13), we will have another different solution provided $W(x, g(a)) \neq W(x, a)$. In fact, if we transform all instances of $a$ in (13) by the map $g$, and use such an invariance property, it follows that we have another solution $(W(x, g(a)), d(g(a)))$ of (13) in addition to $(W(x, a), d(a))$. Inserting each of these pairs into (14) we will obtain in general different partner potentials $\widetilde{V}(x, g(a))$ and $\widetilde{V}(x, a)$ of $V(x, a)$. This also gives an example of the fact that there may exist several different constants $d$ such that we could find a particular solution $W$ of an equation of type (2) or (13) for a fixed $V$.

Another interesting case in which new factorizations can be generated from known ones is when we have a pair of partner potentials $V(x, a)$ and $\widetilde{V}(x, a)$ satisfying the Shape Invariance condition (7), properly understood. In this case this 
condition shows that

$$
V(x, a)=\widetilde{V}\left(x, f^{-1}(a)\right)-R(a),
$$

or in terms of the Hamiltonians,

$$
H(a)=\widetilde{H}\left(f^{-1}(a)\right)-R(a),
$$

which provides an alternative factorization for $H(a)$ :

$$
H(a)=\left(\frac{d}{d x}+W\left(x, f^{-1}(a)\right)\right)\left(-\frac{d}{d x}+W\left(x, f^{-1}(a)\right)\right)+d\left(f^{-1}(a)\right)-R(a),
$$

where it has been used (4) with $A(a)=\frac{d}{d x}+W(x, a)$ and $A^{\dagger}(a)=-\frac{d}{d x}+W(x, a)$. So, had we started only with the potential $V(x, a)$ of this paragraph, we would have been able to find a factorization of $H(a)$ as a product of type $A^{\dagger}(a) A(a)+$ Const. and another as a product $A\left(f^{-1}(a)\right) A^{\dagger}\left(f^{-1}(a)\right)+$ Const., being these last constants different in general.

Of course one could have both situations of the preceding paragraphs at the same time. We shall illustrate them in the next subsection.

\subsection{Illustrative examples}

As a first example we will explain the four-way factorization of the isotropic harmonic oscillator, introduced in Ref. 26, pp. 388-9. In their notation, the potential and Hamiltonian of interest are

$$
V(r, l)=\frac{l(l+1)}{r^{2}}+r^{2}, \quad H(l)=-\frac{d^{2}}{d r^{2}}+V(r, l),
$$

where the independent variable is $r \in(0, \infty)$ and the set of parameters is simply $l$. Their factorization (6) is

$$
H(l)=\left(-\frac{d}{d r}+\frac{l}{r}+r\right)\left(\frac{d}{d r}+\frac{l}{r}+r\right)-(2 l-1),
$$

from where it is suggested that $W(r, l)=\frac{l}{r}+r$. Substituting it in $V(r, l)=$ $W^{2}(r, l)-\frac{W(r, l)}{d r}+d(l)$ we obtain $d(l)=-(2 l-1)$, so (21) is the appropriate version of our (4) as expected. Now, as the potential $V(r, l)$ is invariant under the map $g: l \mapsto-l-1$, we will obtain a new solution $(W(r, g(l)), d(g(l)))=$ $(W(r,-l-1), d(-l-1))$ of the equation

$$
V(r, l)=W^{2}-\frac{d W}{d r}+d
$$

But $W(r, g(l))=W(r,-l-1)=-\frac{l+1}{r}+r$ and $d(g(l))=d(-l-1)=2 l+3$, which is exactly what corresponds to the factorization (4) of Ref. 26 . The factorizations (5) and (7) loc. cit. are related in a similar way; (7) is obtained from (5) by means of the change $g: l \mapsto-l-1$ as well. 
As far as the relation between their factorizations (6) and (5) loc. cit. is concerned, we have already seen that, from their factorization (6), here reproduced as (21), it follows $W(r, l)=\frac{l}{r}+r$, and thus, the corresponding $\widetilde{V}(r, l)$ through (14) is

$$
\widetilde{V}(r, l)=W^{2}(r, l)+\frac{d W(r, l)}{d r}+d(l)=\frac{l(l-1)}{r^{2}}+r^{2}+2 .
$$

Then it is very easy to check that $\widetilde{V}(r, l)=V(r, f(l))+R(f(l))$, where $R(l)=2$ for all $l$, and $f$ is defined either by $f(l)=l-1$ or $f(l)=-l$. We obtain

$$
H(l)=\widetilde{H}(l+1)-R(l), \quad V(r, l)=\widetilde{V}(r, l+1)-R(l),
$$

and

$$
H(l)=\widetilde{H}(-l)-R(l), \quad V(r, l)=\widetilde{V}(r,-l)-R(l),
$$

as well. In this way the factorization (5) of Ref. 26 is achieved.

As a second example we will consider the modified Pöschl-Teller potential, analyzed in an interesting recent article.20 The potential is now

$$
V(x, \alpha, \lambda)=-\alpha^{2} \frac{\lambda(\lambda-1)}{\cosh ^{2} \alpha x},
$$

where $x \in(-\infty, \infty)$ and $\alpha>0, \lambda>1$ are two real parameters.

Two different particular solutions $(W(x, \alpha, \lambda), d(\alpha, \lambda))$ of the Riccati equation

$$
W^{2}-W^{\prime}=V(x, \alpha, \lambda)-d,
$$

have been found in Ref. 20, p. 8450, namely,

$$
\begin{aligned}
& \left(W_{1}(x, \alpha, \lambda), d_{1}(\alpha, \lambda)\right)=\left(-\lambda \alpha \tanh ^{2} \alpha x,-\lambda^{2} \alpha^{2}\right), \\
& \left(W_{2}(x, \alpha, \lambda), d_{2}(\alpha, \lambda)\right)=\left(-(1-\lambda) \alpha \tanh ^{2} \alpha x,-(1-\lambda)^{2} \alpha^{2}\right) .
\end{aligned}
$$

It is clear that the second pair is obtained from the first by means of the parameter transformation $g:(\alpha, \lambda) \mapsto(\alpha, 1-\lambda)$. The reason is that $V(x, \alpha, \lambda)$ is invariant under $g$, or more precisely, its factor $\lambda(1-\lambda)$.

The associated partner potentials $\widetilde{V}(x, \alpha, \lambda)$ obtained using (14), are

$$
\begin{aligned}
& \widetilde{V}_{1}(x, \alpha, \lambda)=W_{1}^{2}(x, \alpha, \lambda)+W_{1}^{\prime}(x, \alpha, \lambda)+d_{1}(\alpha, \lambda)=-\alpha^{2} \frac{\lambda(\lambda+1)}{\cosh ^{2} \alpha x}, \\
& \widetilde{V}_{2}(x, \alpha, \lambda)=W_{2}^{2}(x, \alpha, \lambda)+W_{2}^{\prime}(x, \alpha, \lambda)+d_{2}(\alpha, \lambda)=-\alpha^{2} \frac{(\lambda-1)(\lambda-2)}{\cosh ^{2} \alpha x} .
\end{aligned}
$$

We see that both of the previous functions are just second degree monic polynomials in $\lambda$, with roots spaced one unit, times $-\alpha^{2} / \cosh ^{2} \alpha x$, like $V(x, \alpha, \lambda)$ itself. It is then obvious that a translation of the type $\lambda \mapsto \lambda-b$ or $\lambda \mapsto c-\lambda$ should transform $\widetilde{V}_{1}(x, \alpha, \lambda)$ and $\widetilde{V}_{2}(x, \alpha, \lambda)$ into $V(x, \alpha, \lambda)$. This is in fact so, since $V(x, \alpha, \lambda)=$ $\widetilde{V}_{1}\left(x, \alpha, f^{-1}(\lambda)\right)$, where $f$ is defined either by $f(\lambda)=\lambda-1$ or $f(\lambda)=-\lambda$, and similarly $V(x, \alpha, \lambda)=\widetilde{V}_{2}\left(x, \alpha, f^{-1}(\lambda)\right)$ when $f(\lambda)=\lambda-1$ or $f(\lambda)=2-\lambda$. 
In this way one could propose other different factorizations for the potential $V(x, \alpha, \lambda)$, being able in principle to make a differential operator analysis for this potential similar to what it is done in Ref. 26 for the first example of this Subsection.

\section{Acknowledgments}

A. R. thanks the Spanish Ministerio de Educación y Cultura for a FPI grant, research project PB96-0717. Support of the Spanish DGES (PB96-0717) is also acknowledged.

\section{References}

1. L. Infeld and T.E. Hull, Rev. Mod. Phys. 23, 21 (1951).

2. L.É. Gendenshteïn and I.V. Krive, Soviet Phys. Usp. 28, 645 (1985).

3. J.F. Cariñena, G. Marmo, A.M. Perelomov and M.F. Rañada, Int. J. Mod. Phys. A 13, 4913 (1998).

4. D.J. Fernández, V. Hussin and B. Mielnik, Phys. Lett. A244, 309 (1998).

5. D.J. Fernández and V. Hussin, J. Phys. A: Math. Gen. 32, 3603 (1999).

6. F. Cooper, A. Khare and U.P. Sukhatme, Phys. Rep. 251, 267 (1995).

7. V.B. Matveev and M.A. Salle, Darboux Transformations and Solitons (Springer-Verlag, Berlin and Heidelberg, 1991).

8. A.A. Andrianov, N.V. Borisov, M.V. Ioffe and M.I. Éides, Theor. Math. Phys. 61, 965 (1984).

9. A.A. Andrianov, M.V. Ioffe, F. Cannata and J.P. Dedonder, Int. J. Mod. Phys. A 10, 2683 (1995).

10. V.G. Bagrov and B.F. Samsonov, Theor. Math. Phys. 104, 1051 (1995).

11. D.J. Fernández, Int. J. Mod. Phys. A 12, 171 (1997).

12. A.A. Andrianov, F. Cannata, M.V. Ioffe and D. Nishnianidze, Phys. Lett. A 266, 341 (2000).

13. A.V. Turbiner, Comm. Math. Phys. 118, 467 (1988).

14. M.A. Shifman, Int. J. Mod. Phys. A 4, 2897 (1989).

15. M.A. Shifman and A.V. Turbiner, Comm. Math. Phys. 126, 347 (1989).

16. B. Mielnik, J. Math. Phys. 25, 3387 (1984).

17. D.J. Fernández, Lett. Math. Phys. 8, 337 (1984).

18. M.M. Nieto, Phys. Lett. B 145, 208 (1984).

19. Z. Dongpei, J. Phys. A: Math. Gen. 20, 4331 (1987).

20. J.I. Díaz, J. Negro, L.M. Nieto and O. Rosas-Ortiz, J. Phys. A: Math. Gen. 32, 8447 (1999).

21. L.É. Gendenshteïn, JETP Lett. 38, 356 (1983).

22. A. Stahlhofen, J. Phys. A: Math. Gen. 22, 1053 (1989).

23. R. Montemayor and L.D. Salem, Phys. Rev. A40, 2170 (1987).

24. J.F. Cariñena and A. Ramos, Rev. Math. Phys. 12, to appear (2000). Also: math-ph/9910020

25. J.F. Cariñena and A. Ramos, Int. J. Mod. Phys. A 14, 1935 (1999).

26. D.J. Fernández, J. Negro and M.A. del Olmo, Ann. Phys. (N.Y.) 252, 386 (1996).

27. F. Cooper, J.N. Ginocchio and A. Khare, Phys. Rev. D 36, 2458 (1987).

28. J.F. Cariñena and A. Ramos, J. Phys. A: Math. Gen. 33, 3467 (2000). 